\title{
COMMENTS ON SOME INEQUALITIES OF PEARCE AND PECARIĆ
}

\author{
by HORST ALZER
}

(Received 29th May 1995)

\begin{abstract}
We prove inequalities for convex functions, $L^{p}$ norms, and sums of powers. Our results sharpen recently published inequalities of C. E. M. Pearce and J. E. Pečarić.
\end{abstract}

1991 Mathematics subject classification: $26 \mathrm{D} 15$.

\section{Introduction}

The study of singular measures led G. Brown [1] in 1988 to several interesting new inequalities involving polynomials and fractional powers. These inequalities were reexamined by A. W. Kemp [4] in 1992. In order to shorten the proof of Brown's main theorem, Kemp applied a specific inequality for convex functions which was generalized by C. E. M. Pearce and J. E. Pečarić. Their result says:

Theorem A. (Pearce and Pečarić, [5]). Let $a, b, s_{i}$ and $t_{i}(i=0,1,2)$ be positive real numbers with $a / s_{i}+b / t_{i}=1(i=0,1,2)$ and $s_{1}<s_{0}<s_{2}$. If $f, g:(0, \infty) \rightarrow \mathbb{R}$ are convex functions, then

$$
\frac{f\left(s_{0}\right)}{s_{0}}+\frac{g\left(t_{0}\right)}{t_{0}} \leq \max _{i=1,2}\left(\frac{f\left(s_{i}\right)}{s_{i}}+\frac{g\left(t_{i}\right)}{t_{i}}\right)
$$

In 1989, G. Brown and L. Shepp [2] presented two "key lemmas" [6, p. 60] which are useful in calculations with fractal sets. They proved two inequalities for $L^{p}$ norms and sums of powers which are of the same type of inequalities as given in Theorem A. Recently, C. E. M. Pearce and J. E. Pečaric have published the following extensions.

Theorem B. (Pearce and Pečarić, [6]). Let the positive real numbers $a, b, s_{i}$ and $t_{i}(i=0,1,2)$ satisfy $a / s_{i}+b / t_{i}=1(i=0,1,2)$ and $s_{1}<s_{0}<s_{2}$.

(i) If $\|\cdot\|_{p}$ denotes the $L^{p}$ norm of a real-valued function, then 


$$
\|f\|_{s_{0}}\|g\|_{t_{0}} \leq \max _{i=1,2}\left(\|f\|_{s_{i}}\|g\|_{t_{i}}\right)
$$

provided that all quantities exist.

(ii) Let $x=\left(x_{i}\right), u=\left(u_{i}\right)(i=1, \ldots, n)$ and $y=\left(y_{j}\right), v=\left(v_{j}\right)(j=1, \ldots, m)$ be sequences of positive real numbers. If

$$
S_{n}^{[l]}(x, u)=\left(\sum_{i=1}^{n} u_{i} x_{i}^{\ell}\right)^{1 / t}
$$

then

$$
S_{n}^{\left[s_{0}\right]}(x, u) S_{m}^{\left[t_{0}\right]}(y, v) \leq \max _{i=1.2}\left(S_{n}^{\mid s]}(x, u) S_{m}^{\left[t_{i} \mid\right.}(y, v)\right)
$$

If $\sum_{i=1}^{n} u_{i}=1$, then $S_{n}^{[l]}(x, u)$ is the weighted power mean of order $t$. And, if $u_{i}=1$ $(i=1, \ldots, n)$, then we have the sum of order $t$. Many remarkable properties of these power sums can be found in the monograph [3].

It is the main purpose of this paper to present refinements of Theorem $A$ and Theorem B. A central role in our proofs plays an inequality for arithmetic means which might be of independent interest.

\section{An inequality for arithmetic means}

The following inequality sharpens $A_{n} \leq \max _{1 \leq i \leq n} a_{i}$, where $A_{n}$ denotes the weighted arithmetic mean of the real numbers $a_{1}, \ldots, a_{n}$.

Theorem 1. Let $p_{i}(i=1, \ldots, n ; n \geq 2)$ be positive real numbers with $\sum_{i=1}^{n} p_{i}=1$. Then we have for all real numbers $a_{1}, \ldots, a_{n}$ :

$$
\frac{p}{n-1} \sum_{1 \leq i<j \leq n}\left(\sqrt{\left|a_{i}\right|}-\sqrt{\left|a_{j}\right|}\right)^{2} \leq \max _{1 \leq i \leq n} a_{i}-\sum_{i=1}^{n} p_{i} a_{i}
$$

where

$$
p=\min _{i \leq i \leq n} p_{i}
$$

Proof. Let 


$$
S\left(a_{1}, \ldots, a_{n}\right)=\max _{1 \leq i \leq n} a_{i}-\sum_{i=1}^{n} p_{i} a_{i}-\frac{p}{n-1} \sum_{1 \leq i<j \leq n}\left(\sqrt{\left|a_{i}\right|}-\sqrt{\left|a_{j}\right|}\right)^{2} .
$$

Without loss of generality we may assume that $a_{n} \leq a_{n-1} \leq \ldots \leq a_{1}$. We consider two cases.

Case 1: $0 \leq a_{n}$ or $a_{1} \leq 0$.

Then we have

$$
\begin{aligned}
\sum_{1 \leq i<j \leq n}\left(\sqrt{\left|a_{i}\right|}\right. & \left.-\sqrt{\left|a_{j}\right|}\right)^{2} \\
& =(n-1) \sum_{i=1}^{n}\left|a_{i}\right|-2 \sum_{1 \leq i<j \leq n} \sqrt{\left|a_{i} a_{j}\right|} \\
& \leq(n-1) \sum_{i=1}^{n}\left|a_{i}\right|-2 \sum_{1 \leq i<j \leq n} \min \left(\left|a_{i}\right|,\left|a_{j}\right|\right) \\
& =\sum_{i=1}^{n}(n+1-2 i) a_{i} .
\end{aligned}
$$

We set $q_{i}=p_{i}+[(n+1-2 i) /(n-1)] p(i=1, \ldots, n)$; then we have $q_{i} \geq 0(i=1, \ldots, n)$ and $\sum_{i=1}^{n} q_{i}=1$. Hence, we conclude from (2.2):

$$
S\left(a_{1}, \ldots, a_{n}\right) \geq a_{1}-\sum_{i=1}^{n} q_{i} a_{i} \geq 0 .
$$

Case 2: $\quad a_{n} \leq \cdots \leq a_{k+1} \leq 0 \leq a_{k} \leq \cdots \leq a_{1}$, where $k \in\{1, \ldots, n-1\}$. Then

$$
S\left(a_{1}, \ldots, a_{n}\right)=a_{1}-\sum_{i=1}^{n} p_{i} a_{i}-p \sum_{i=1}^{n}\left|a_{i}\right|+\frac{2 p}{n-1} \sum_{1 \leq i<j \leq n} \sqrt{\left|a_{i} a_{j}\right|}
$$

We define for $r=1, \ldots, k$ and $a>0$ :

$$
S_{r}(a)=S\left(a, \ldots, a, a_{r+1}, \ldots, a_{n}\right)
$$

Differentiation yields 


$$
\begin{aligned}
S_{r}^{\prime}(a) & =1-\sum_{i=1}^{r} p_{i}-p r+\left(\frac{(r-1) r}{2}+\frac{r}{2 \sqrt{a}} \sum_{i=r+1}^{n} \sqrt{\left|a_{i}\right|}\right) \frac{2 p}{n-1} \\
& \geq 1-\sum_{i=1}^{r} p_{i}-p r+\frac{(r-1) r p}{n-1} \\
& \geq\left(n-2 r+\frac{(r-1) r}{n-1}\right) p \\
& \geq 0,
\end{aligned}
$$

where the last inequality follows from $1 \leq r \leq n-1$. Hence, $S_{r}$ is increasing on $[0, \infty)$. Using the monotonicity of $S_{r}$ and $S_{m-l}\left(a_{m}\right)=S_{m}\left(a_{m}\right)(m=2, \ldots, k)$, we get

$$
\begin{aligned}
S\left(a_{1}, \ldots, a_{n}\right) & =S_{1}\left(a_{1}\right) \geq S_{1}\left(a_{2}\right)=S_{2}\left(a_{2}\right) \geq S_{2}\left(a_{3}\right) \\
& \geq \cdots \geq S_{k}\left(a_{k}\right) \geq S_{k}(0) \geq \sum_{i=k+1}^{n}\left(p-p_{i}\right) a_{i} \geq 0 .
\end{aligned}
$$

This completes the proof of Theorem 1.

Remarks. (1) Let $p_{k}=\min _{1 \leq i \leq n} p_{i}$; if we set $a_{i}=1(i=1, \ldots, n ; i \neq k)$ and $a_{k}=0$, then we have

$$
\left(\max _{1 \leq i \leq n} a_{i}-\sum_{i=1}^{n} p_{i} a_{i}\right) / \sum_{1 \leq i<j \leq n}\left(\sqrt{\left|a_{i}\right|}-\sqrt{\left|a_{j}\right|}\right)^{2}=\frac{p_{k}}{n-1}
$$

Hence, in $(2.1)$ the constant factor $[1 /(n-1)] \min _{1 \leq i \leq n} p_{i}$ cannot be replaced by a greater number.

(2) If we set $0<a_{1}=\cdots=a_{n-1}<a_{n}=1$, then we obtain

$$
\begin{aligned}
& \lim _{a_{1} \rightarrow 1}\left(\max _{1 \leq i \leq n} a_{i}-\sum_{i=1}^{n} p_{i} a_{i}\right) / \sum_{1 \leq i<j \leq n}\left(\sqrt{\left|a_{i}\right|}-\sqrt{\left|a_{j}\right|}\right)^{2} \\
& \quad=\lim _{a_{1} \rightarrow 1} \frac{1-p_{n}}{n-1} \frac{1+\sqrt{a_{1}}}{1-\sqrt{a_{1}}}=\infty .
\end{aligned}
$$

This implies that there does not exist a converse of inequality (2.1), that is, there does not exist a constant $c\left(p_{1}, \ldots, p_{n}\right)$ such that the inequality

$$
\max _{1 \leq i \leq n} a_{i}-\sum_{i=1}^{n} p_{i} a_{i} \leq c\left(p_{1}, \ldots, p_{n}\right) \sum_{1 \leq i<j \leq n}\left(\sqrt{\left|a_{i}\right|}-\sqrt{\left|a_{j}\right|}\right)^{2}
$$

holds for all real numbers $a_{1}, \ldots, a_{n}$. 


\section{An inequality for convex functions}

The following proposition provides a sharpening of inequality (1.1).

Theorem 2. Let $a, b, s_{i}$ and $t_{i}(i=0,1,2)$ be positive real numbers with $a / s_{i}+b / t_{i}=1$ $(i=0,1,2)$ and $s_{1}<s_{0}<s_{2}$, and let $f, g:(0, \infty) \rightarrow \mathbb{R}$ be convex functions. Then

$$
h_{0} \leq \max \left(h_{1}, h_{2}\right)-\alpha\left[\sqrt{\left|h_{1}\right|}-\sqrt{\left|h_{2}\right|}\right]^{2}
$$

where

$$
h_{i}=\frac{f\left(s_{i}\right)}{s_{i}}+\frac{g\left(t_{i}\right)}{t_{i}} \quad(i=0,1,2)
$$

and

$$
\alpha=\min \left(\frac{\left(s_{2}-s_{0}\right) s_{1}}{\left(s_{2}-s_{1}\right) s_{0}}, \frac{\left(s_{0}-s_{1}\right) s_{2}}{\left(s_{2}-s_{1}\right) s_{0}}\right) \in(0,1) .
$$

Proof. We follow the method of proof given in [5]. There exists a real number $\delta \in(0,1)$ such that

$$
s_{0}=\delta s_{1}+(1-\delta) s_{2}
$$

We set $\eta=\delta\left(s_{1} / s_{0}\right)\left(t_{0} / t_{1}\right)$; then we obtain

$$
\begin{aligned}
\eta+(1-\delta) \frac{s_{2}}{s_{0}} \frac{t_{0}}{t_{2}} & =\frac{t_{0}}{s_{0}}\left(\delta \frac{s_{1}}{t_{1}}+(1-\delta) \frac{s_{2}}{t_{2}}\right) \\
& =\frac{b}{s_{0}-a}\left(\delta \frac{s_{1}-a}{b}+(1-\delta) \frac{s_{2}-a}{b}\right) \\
& =\frac{\delta s_{1}+(1-\delta) s_{2}-a}{s_{0}-a} \\
& =1
\end{aligned}
$$

which leads to

$$
t_{0}=\eta t_{1}+(1-\eta) t_{2}, \quad \eta \in(0,1)
$$

Applying Jensen's inequality we get from (3.1) and (3.2):

$$
f\left(s_{0}\right) \leq \delta f\left(s_{1}\right)+(1-\delta) f\left(s_{2}\right)
$$


and

$$
g\left(t_{0}\right) \leq \eta g\left(t_{1}\right)+(1-\eta) g\left(t_{2}\right)
$$

Since $\eta\left(t_{1} / t_{0}\right)=\delta\left(s_{1} / s_{0}\right)$ and $(1-\eta)\left(t_{2} / t_{0}\right)=(1-\delta)\left(s_{2} / s_{0}\right)$, we conclude from (3.3), (3.4) and (2.1) (with $n=2$ ):

$$
\begin{aligned}
h_{0} & =\frac{f\left(s_{0}\right)}{s_{0}}+\frac{g\left(t_{0}\right)}{t_{0}} \\
& \leq \delta \frac{s_{1}}{s_{0}}\left(\frac{f\left(s_{1}\right)}{s_{1}}+\frac{g\left(t_{1}\right)}{t_{1}}\right)+(1-\delta) \frac{s_{2}}{s_{0}}\left(\frac{f\left(s_{2}\right)}{s_{2}}+\frac{g\left(t_{2}\right)}{t_{2}}\right) \\
& =\delta \frac{s_{1}}{s_{0}} h_{1}+(1-\delta) \frac{s_{2}}{s_{0}} h_{2} \\
& \leq \max \left(h_{1}, h_{2}\right)-\min \left(\delta \frac{s_{1}}{s_{0}},(1-\delta) \frac{s_{2}}{s_{0}}\right)\left[\sqrt{\left|h_{1}\right|}-\sqrt{\left|h_{2}\right|}\right]^{2} \\
& =\max \left(h_{1}, h_{2}\right)-\alpha\left[\sqrt{\left|h_{1}\right|}-\sqrt{\left|h_{2}\right|}\right]^{2}
\end{aligned}
$$

\section{Inequalities for $L^{p}$ norms and power sums}

We establish the following refinements of inequalities (1.2) and (1.3).

Theorem 3. Let $a, b, s_{i}$ and $t_{i}(i=0,1,2)$ be positive real numbers such that $a / s_{i}+b / t_{i}=1(i=0,1,2)$ and $s_{1}<s_{0}<s_{2}$. Furthermore, let

$$
\alpha=\min \left(\frac{\left(s_{2}-s_{0}\right) s_{1}}{\left(s_{2}-s_{1}\right) s_{0}}, \frac{\left(s_{0}-s_{1}\right) s_{2}}{\left(s_{2}-s_{1}\right) s_{0}}\right) .
$$

(i) Assuming that all quantities exist, we have

$$
P_{0} \leq \max \left(P_{1}, P_{2}\right)-\alpha\left[\sqrt{P_{1}}-\sqrt{P_{2}}\right]^{2}
$$

where

$$
P_{i}=\|f\|_{s_{i}}\|g\|_{t_{i}} \quad(i=0,1,2)
$$

(ii) If $x=\left(x_{i}\right), u=\left(u_{i}\right)(i=1, \ldots, n)$ and $y=\left(y_{j}\right), v=\left(v_{j}\right)(j=1, \ldots, m)$ are sequences of positive real numbers, then

$$
Q_{0} \leq \max \left(Q_{1}, Q_{2}\right)-\alpha\left[\sqrt{Q_{1}}-\sqrt{Q_{2}}\right]^{2}
$$


where

$$
\begin{aligned}
& Q_{i}=S_{n}^{\left[s_{i}\right]}(x, u) S_{m}^{\left[i_{i}\right]}(y, v) \quad(i=0,1,2) \quad \text { and } \\
& S_{n}^{[l]}(x, u)=\left(\sum_{i=1}^{n} u_{i} x_{i}^{t}\right)^{1 / t}
\end{aligned}
$$

Proof. (i) Let

$$
\delta=\frac{s_{2}-s_{0}}{s_{2}-s_{1}} \quad \text { and } \quad \eta=\delta \frac{s_{1}}{s_{0}} \frac{t_{0}}{t_{1}}
$$

Then, as shown in the proof of Theorem 2 , we have $\delta, \eta \in(0,1)$ and

$$
s_{0}=\delta s_{1}+(1-\delta) s_{2} \quad \text { and } \quad t_{0}=\eta t_{1}+(1-\eta) t_{2}
$$

Applying Hölder's inequality for integrals we obtain

$$
\|f\|_{s_{0}}^{s_{0}} \leq\|f\|_{s_{1}}^{\delta s_{1}}\|f\|_{s_{2}}^{(1-\delta) s_{2}}
$$

and

$$
\|g\|_{t_{0}}^{t_{0}} \leq\|g\|_{t_{1}}^{\eta t_{1}}\|g\|_{t_{2}}^{(1-\eta) t_{2}}
$$

which implies, since $\eta\left(t_{1} / t_{0}\right)=\delta\left(s_{1} / s_{0}\right)$ and $(1-\eta)\left(t_{2} / t_{0}\right)=(1-\delta)\left(s_{2} / s_{0}\right)$,

$$
\begin{aligned}
P_{0} & =\|f\|_{s_{0}}\|g\|_{t_{0}} \\
& \leq\left(\|f\|_{s_{1}}\|g\|_{t_{1}}\right)^{\delta s_{1} / s_{0}}\left(\|f\|_{s_{2}}\|g\|_{t_{2}}\right)^{(1-\delta) s_{2} / s_{0}} \\
& =P_{1}^{\delta s_{1} / s_{0}} P_{2}^{(1-\delta) s_{2} / s_{0}} .
\end{aligned}
$$

From (4.5), the arithmetic mean-geometric mean inequality $a^{p} b^{q} \leq p a+q b(a, b \geq 0$; $p, q>0, p+q=1$ ) and (2.1) (with $n=2$ ) we get

$$
\begin{aligned}
P_{0} & \leq \delta \frac{s_{1}}{s_{0}} P_{1}+(1-\delta) \frac{s_{2}}{s_{0}} P_{2} \\
& \leq \max \left(P_{1}, P_{2}\right)-\min \left(\delta \frac{s_{1}}{s_{0}},(1-\delta) \frac{s_{2}}{s_{0}}\right)\left[\sqrt{P_{1}}-\sqrt{P_{2}}\right]^{2} \\
& =\max \left(P_{1}, P_{2}\right)-\alpha\left[\sqrt{P_{1}}-\sqrt{P_{2}}\right]^{2} .
\end{aligned}
$$

(ii) If $\delta$ and $\eta$ are given by (4.3), then we conclude from (4.4) and Hölder's 
inequality for sums that

$$
\left(S_{n}^{\left[s_{0}\right]}(x, u)\right)^{s_{0}} \leq\left(S_{n}^{\left[s_{1}\right]}(x, u)\right)^{\delta s_{1}}\left(S_{n}^{\left[s_{2}\right]}(x, u)\right)^{(1-\delta) s_{2}}
$$

and

$$
\left(S_{m}^{\left[t_{0}\right]}(y, v)\right)^{t_{0}} \leq\left(S_{m}^{\left[t_{1}\right]}(y, v)\right)^{\eta t_{1}}\left(S_{m}^{\left[t_{2}\right]}(y, v)\right)^{(1-\eta) t_{2}}
$$

This leads to

$$
\begin{aligned}
Q_{0} & =S_{n}^{\left[s_{0}\right]}(x, u) S_{m}^{\left[t_{0}\right]}(y, v) \leq\left(S_{n}^{\left[s_{1}\right]}(x, u) S_{m}^{\left[t_{1}\right]}(y, v)\right)^{\delta s_{1} / s_{0}}\left(S_{n}^{\left[s_{2}\right]}(x, u) S_{m}^{\left[t_{2}\right]}(y, v)\right)^{(1-\delta) s_{2} / s_{0}} \\
& =Q_{1}^{\delta s_{1} / s_{0}} Q_{2}^{(1-\delta) s_{2} / s_{0}}
\end{aligned}
$$

which implies, as shown in (i), that

$$
Q_{0} \leq \max \left(Q_{1}, Q_{2}\right)-\alpha\left[\sqrt{Q_{1}}-\sqrt{Q_{2}}\right]^{2}
$$

Acknowledgement. I thank the referee for helpful suggestions.

\section{REFERENCES}

1. G. Brown, Some inequalities that arise in measure theory, J. A ustral. Math. Soc. Ser. A 45 (1988), 83-94.

2. G. Brown and L. Shepp, A convolution inequality, in: Contributions to Probability and Statistics, Essays in Honour of I. Olkin (Springer, New York, 1989), 51-57.

3. P. S. Bullen, D. S. Mitrinović, and P. M. Vasić, Means and Their Inequalities (D. Reidel, Dordrecht, 1988).

4. A. W. Kemp, Certain inequalities involving fractional powers, J. Austral. Math. Soc. Ser. A 53 (1992), 131-136.

5. C. E. M. Pearce and J. E. PeČarić, An inequality for convex functions, J. Math. Anal. Appl. 183 (1994), 523-527.

6. C. E. M. Pearce and J. E. PeČarić, On two lemmas of Brown and Shepp having application to sum sets and fractals, J. Austral. Math. Soc. Ser. B 36 (1994), 60-63.

MORSBacher STR. 10

51545 WALDBRÖL

GERMANY 